\title{
Advances and prospects in glucose assay technology
}

\author{
D .A . G ough, J .C. A rmour, D.A . B aker \\ Department of Bioengineering, University of California, San Diego, USA
}

Summary The evolution of glucose assay methods has been central to the development of present therapies for diabetes mellitus. However, new blood glucose assay capabilities, such as convenient and truly continuous monitoring, are now needed to foster a new era of close blood glucose control. The principles and present status of glucose sensors being developed that may fill these requirements are reviewed here. [Diabetologia (1997) 40: S102-S107]

Keywords Implantable glucose sensors
Glucose assay has long been central to therapy of diabetes mellitus. In the spirit of this occasion commemorating the 75th Anniversary of the Discovery of Insulin, we review here some of the important advances in glucose assay methodology, give our opinions on the present status of glucose assay technologies, and speculate on potential advances in the near future. Some aspects of this review have recently been published elsewhere [1].

\section{A brief history}

Glucose assays have been central to the treatment of diabetes from the early days of treatment. The discovery of the effect of insulin at the biochemical level was made possible by an early glucose assay based on the chromogenic reduction of a copper solution by glucose [2]. This assay and its successive modifications were not completely specific for glucose in the presence of other reducing sugars and required careful preparation of relatively large samples, but were nevertheless useful for laboratory assay of glucose in blood and urine. In the following years, clinical

Corresponding author: D. A. Gough, Department of Bioengineering, University of California, San Diego, La Jolla, CA 92093-0412, USA glucose assay methodology evolved rapidly, incorporating enzymes for specificity, requiring less sample preparation, smaller volumes, and becoming fully automated [2].

Qualitative tests for glucose were later developed based on glucose oxidase and reagents impregnated in paper strips. This advance facilitated patient involvement in glucose assay and the approach was used widely for urine glucose determinations because of its convenience, even though the assay was originally considered less reliable than blood assays. In the past decade, this technology has advanced dramatically in conjunction with the development of home glucometer instrumentation, to the point of providing quantitative, convenient and inexpensive blood glucose determination. The evolution of this technology represents a revolutionary improvement in the management of diabetes, as widespread, frequent and quantitative blood glucose testing is now possible, leading to more appropriate insulin administration schedules and closer involvement of the patient in therapy [3].

\section{D esired new features}

The results of the Diabetes Control and Complications Trial [4] suggest the value of close blood glucose control in reducing the complications of 
diabetes. These important results offer new hope for people with diabetes, but also pose a difficult problem: blood glucose control is presently based on blood glucose assay which, with available methodology, still requires frequent blood sampling with some associated degree of inconvenience. Recent studies suggest that some people with diabetes may choose not to strive for close blood glucose control because of the obtrusiveness of blood sampling and assay [5].

Even with the most assiduous use of the present glucose assay methodology however, it may still not be feasible to maintain close blood glucose control. This is because the frequency of blood sampling that is acceptable to most patients is nevertheless infrequent compared to the rate of blood glucose fluctuations. (Lags due to glucose correction mechanisms, such as insulin administration and uptake, may further interfere with the achievement of close control.) In addition, irregular, initiative-based sampling cannot be relied upon to detect spontaneous hypoglycaemia. Blood sampling provides a discrete rather than continuous blood glucose record, and is not sufficiently frequent and regular to serve as a warning of rapid blood glucose imbalances.

As useful as the present glucose assay technologies have become, an important feature that remains to be incorporated is the capability of truly continuous monitoring. This would permit therapeutic options that are not at present available. For example, continuous glucose sensing would be advantageous in the detection and management of hypoglycaemia, which would be particularly beneficial for hypoglycaemia-insensitive patients [6]. Continuous glucose sensing would also allow early detection of hyperglycaemia and provide a basis for insulin administration at more appropriate dosages and timing, or eventually, for automatic insulin delivery from a pump. A continuous glucose sensor device could also be employed beneficially in parallel with other existing or potential forms of insulin replacement, such as transplantation or hybrid islet devices, even though the sensor signal would not be coupled to insulin delivery from these sources. Nevertheless, new continuous sensing technologies will still have to be unobtrusive and more convenient than previous discrete sensing approaches to achieve widespread application.

Regardless of the above, the fundamental requirement of any assay method is accuracy. The sensor signal must indicate the instantaneous blood glucose concentration within a specified error. The reported concentration must be reliable and the user must not have to judge its accuracy. Further, the signal should not require frequent recalibration by the user. The development of a stable, highly accurate and continuous sensor that is acceptable to patients is a significant challenge.

\section{Physical principles for continuous sensors}

Although there are many physical principles for measuring glucose under ideal conditions, few show promise for this challenging application. Sensor designs and their respective potential advantages and disadvantages have recently been reviewed [1]. The approaches with most promise are mentioned here.

One possible approach is the hydrogen peroxidebased enzyme electrode sensor [7-14]. This design has a membrane containing immobilized glucose oxidase coupled to a peroxide-sensitive catalytic anode. Glucose and oxygen diffuse into the membrane where an enzymatic reaction occurs in which peroxide is produced. The peroxide diffuses through an underlying porous membrane to the anode, where it is electrochemically oxidized to produce the signal current. The external component of the current passes from the anode, through the membranes and tissue or blood, to a nearby cathode. The main difficulties associated with this type of sensor include electrochemical interference $[15,16]$, accounting for local oxygen variability $[12,13]$, and peroxide-mediated enzyme inactivation [17].

An alternative is the oxygen-based enzyme electrode sensor [18-21], which has a membrane containing immobilized glucose oxidase coupled to a membrane-covered electrochemical oxygen sensor. Glucose and oxygen diffuse into the membrane and react, resulting in a reduction of the amount of oxygen that would otherwise be detected by the oxygen sensor. The signal current is subtracted from that of a similar reference oxygen sensor without the enzyme and a glucose-dependent difference current results. This sensor is unaffected by electrochemical interference from endogenous species, is transparent to oxygen variations, and can include co-immobilized catalase to reduce peroxide-mediated enzyme inactivation, but may be slightly more difficult to produce.

Non-invasive sensor concepts have also received considerable attention in recent years. The premise is that light in the near infrared or other region of the spectrum that has some sensitivity to glucose is beamed on a relatively transparent region of tissue such as the finger web [22]. The transmitted or reflected light signal is processed by mathematical filtering techniques to maximize any aspects of the signal that may show some correlation to blood glucose samples obtained at the time of the observation. The overwhelming problem of this approach is the lack of adequate selectivity for glucose. There is substantial chemical interference from many biological molecules, as well as physical interference from tissue structures, temporal and spatial variations in perfusion, and several optical effects. In addition, there is a fundamental reservation about calibration of the device: the retrospective correlations between signal and blood glucose concentration described above 
change with time and are not useful for real time monitoring. These issues must be resolved by systematic mechanistic studies before further industrial development can be justified. The best hope for this approach may be to establish a separate qualitative relationship for each user that detects large glucose changes. However, even this degree of response will be very difficult to validate at best and may lead to a sensor that is of minimal clinical value. Despite substantial industrial investment, media attention, and considerable enthusiasm on the part of proponents, prospects for implementation of a non-invasive glucose sensor in the near future are not encouraging.

Several approaches to non-invasive transdermal extraction of tissue fluids containing glucose are being considered as alternatives to blood collection. Tissue fluid extraction by hydrostatic pressure, electrophoresis, ultrasound, or other means may be feasible, but it must be demonstrated that these methods have advantages over blood collection. Moreover, it remains to be shown that glucose in these extracts correlates with blood glucose, that the dynamic response is adequate, and that the extraction techniques can be convenient and do not cause adverse tissue reactions, especially when used over the long term. Due to the time lags associated with fluid collection, these methods may be limited to discrete, infrequent determinations.

Other concepts based on mediated enzyme electrode sensors, microdialysis, electrochemical sensors, and monitoring physiological effects of glucose have been described in detail elsewhere [1]. In our view, these approaches are less likely to yield practical sensors in the near future.

\section{Potential configurations of implantable sensors}

When ultimately implemented, the implantable glucose sensor may take several configurations [1]:

A short-term intravenous sensor similar to a catheter would be used in hospitalized patients for up to $72 \mathrm{~h}$. This application may be useful in diagnosis and glycaemic stabilization, management of ketoacidosis, surgery and recovery, women in labour with glucose instabilities, intensive care of certain neonates, and similar monitoring situations.

A short-term subcutaneous sensor would be inserted into subcutaneous tissues of non-hospitalized patients for periods of several days. The sensor would be connected percutaneously to an external data storage and display device in the form of a wrist watch or belt-mounted beeper. This type of sensor will be attractive if it is inexpensive, does not require frequent recalibration, and can be considered disposable. Industry has devoted attention to this application at the expense of other implant applications because of its perceived commercial potential.
A long-term sensor would be implanted either intravenously or in tissues for periods of up to 1 year. This sensor could be coupled to an implanted telemetry system that transmits information to an external receiver and percutaneous components would not be necessary. This configuration will have to be easily inserted and retrieved with minimal surgery. Recalibration may be acceptable if simple and infrequent (say, monthly). In each of these configurations, the sensor could be used either as a monitor or as part of an automatic feedback-controlled insulin delivery system.

\section{Implant studies}

Short-term subcutaneous implants. The short-term subcutaneous sensor is the most difficult application. The peroxide-based sensor has been fabricated in the form of a needle and implanted in dogs [23, 24], rats $[10,14]$ and pigs [25]. The sensors typically remained in place for several days and signals were recorded in response to intravenous glucose challenges. The sensitivity and baseline of each sensor output were independently adjusted after each experiment. Anaesthesia was used during testing in most cases. The most common experimental protocol was to determine how closely statistical averages of signals at discrete times from various sensors correlated with statistical averages of blood glucose concentration. Responses of sensors that did not meet expectations were often ignored. Due to the emphasis on statistical correlation, there was no attempt to interpret the signal of individual sensors, although an interpretation of this kind is necessary for actual application of the sensor. Little understanding of the factors that affect glucose transport to implanted sensors was revealed. These studies show that subcutaneous sensors can sometimes produce a response to blood glucose challenges under ideal conditions, but it remains unclear whether the response can be useful for glucose monitoring.

There has also been an attempt to use the peroxide-based sensor as a long-term implant in dog subcutaneous tissue [13]. The sensor and a telemetry unit [26] were implanted for periods of up to 90 days. The sensor signal decayed continuously over the implant period and the sensor required recalibration prior to each recording session. No determination was made of the maximal period the sensor could operate accurately without recalibration, although this was apparently at most only a few days. Thus, even though the implant remained in place for up to 90 days, it cannot be considered a reliable, functioning sensor for that period. It would not be feasible for such a sensor to accurately indicate spontaneous glucose fluctuations or be employed for purposes of continuous control. The authors reported that the range of sensitivity to glucose decreased significantly over the implant period due to an increasing oxygen 
limitation, even after recalibration. This may have been caused by enzyme inactivation, progressive electrochemical interference, or an advancing tissue reaction. The important question of whether the limitations were due to sensor design, tissue remodelling, or to local physiologic phenomena was not considered. The many questions raised by this study remain to be addressed.

The peroxide-based sensor in the form of a fine needle has been used as a short-term subcutaneous implant in humans $[9,12,27-29]$. In these studies, sensors were connected by percutaneous leads to wearable instrumentation and there was no need for local anaesthesia. Statistical correlations were reported between groups of sensor responses and averaged blood glucose concentrations determined by a standard method. As judged by in vitro characterization prior to implantation, sensors typically had a rapid response to concentration changes and were linear over clinically useful ranges. Most studies noted a substantial decay in signal over the several day implant period, but the sensor responses were typically adjusted after the experiment to correspond to actual blood glucose values. One group [29] employed a rapid recalibration device that adjusted readings during use based on glucose values determined by frequent blood sampling. Extensive histological studies of the sensor environment were not feasible in humans.

Although most investigators interpreted these results as highly promising, in our view, there are a number of reservations. First, there is the problem of decay in sensitivity over the implant period. This has led to the use of retrospective calibration, in which the sensor sensitivity is adjusted after the experiment to match independently determined steady-state blood glucose values. In addition, the actual values of the signal without recalibration are not often reported, making it difficult to compare actual rates of signal decay. The correlation between steady-state blood glucose and retrospectively adjusted signal conveys the impression that the sensor can be useful in real time, but in fact monitoring in real time cannot be based on retrospective calibration unless there is a highly reproducible pattern of signal decay, which has yet to be established. Improved means of calibration must be developed.

Other reservations pertain to the tissue structure around the sensor. The technique of sensor insertion may be of crucial importance and there must be means of knowing whether a functional placement has been achieved. Trauma associated with sensor placement leads to tissue inflammation and the wound healing process, which may interfere with stabilization of the sensor signal. The insertion must not cause significant bleeding or lead to the formation of a reservoir of tissue fluid around the sensor. The resulting local hyperaemia must stabilize rapidly after insertion and give rise to a stationary mass-transfer field in order to expect reliable readings. Given these considerations, it is unlikely that the sensor can be inserted and used shortly thereafter to confidently administer medication. There is a need for better understanding of the effects, time course, and reproducibility of trauma associated with sensor insertion.

Data selection in these studies is also problematic. As it is not possible to present all results, representative results must be selected. However, results selected with the intent of demonstrating that the sensor is 'working' often do not indicate the range of responses typically obtained, which would be useful for a deeper understanding. Furthermore, reports of the percentage of apparently functional sensor implants do not convey the troubling inability to predict whether a specific sensor that is functional in vitro will give meaningful signals when implanted subcutaneously. Data selection practices and incomplete description of results may unintentionally convey the impression that favourable results are more common than is actually the case.

In addition to issues of calibration, tissue stabilization, and data selection, it is our view that there is inadequate definition of the relationship between the signal of a sensor implanted in tissues and actual blood glucose values. A simple, stable proportionality that applies with confidence in all situations probably does not exist. If a generally useful relationship is found, it will be more complicated than presently appreciated. The glucose signal is affected substantially by dynamic physiological aspects of the sensor environment and changes in the environment with time. For example, microvascular perfusion of the site and therefore delivery of glucose to the sensor may be strongly influenced by transient events such as sympathetic stimulation, thermal effects, and hydrostatic variations due to posture, movement, etc. Unfortunately, most research to date has been directed at finding a simple statistical correlation between the signal and blood glucose concentration, rather than understanding the role of relevant physiologic phenomena and developing a deterministic model of glucose transport in living tissue that can be used in a predictive fashion. Moreover, in many cases blood sampling has not been sufficiently frequent to clearly define blood glucose fluctuations. More carefully conceived research is needed to establish the generality of signal-blood glucose relationships.

There is likely to be no consensus on the ultimate promise of short-term tissue implant applications until these controversies are resolved. These questions have led to the most important difference of opinion - whether human trials of short-term subcutaneous sensors are presently justified. Early studies in humans have emphasized correlative, trail-and-error observations, rather than mechanistic research. Many questions can be more effectively addressed at present with animal studies. There is an urgent need for directed basic studies that lead to a better 
understanding of the sensor response in the tissue environment before the results of clinical trials can be interpreted. It will not be possible to effectively address deficiencies in response without a better basic understanding. Support for basic studies of this type does not fall in the domain of industrial research and must come from public sources. In all, there is much to be learned before the subcutaneous sensor can be operated confidently.

Short-term intravenous sensor. Technically, the shortterm intravenous sensor for in-patient use is much more straightforward. The sensor could be coupled to an intravenous fluid flush system and may have no greater propensity for complications than the intravenous catheters presently in use. Both the oxygen-based and peroxide-based sensors have been operated intravenously in animals $[10,30]$. No further basic research is needed and this is the most direct route to clinical application of an implantable sensor, although the demand may be small relative to other potential applications.

L ong-term intravenous sensor. The oxygen-based sensor has been implanted intravenously in six dogs for periods of 7 to 108 days [20]. The glucose sensor was at the tip of a cylinder $2 \mathrm{~mm}$ outer diameter by approximately $30 \mathrm{~cm}$ in total length. The sensor and a similar oxygen reference sensor were advanced into the jugular vein so that the tips were positioned in the centre of the superior vena cava several centimeters above the entrance of the right atrium. The sensor leads were connected to a telemeter and instrumentation unit [31] implanted subcutaneously. No systemic anticoagulation was needed. The sensitivity to glucose, determined before implantation, during use, and after explantation, was not substantially altered by longterm implantation [20] and there was no need for recalibration during the entire period of the implantation. The experiments were not limited by immobilized enzyme lifetime, oxygen deficit, oxygen sensor instability, chemical interference or biological incompatibility. These results demonstrate that this glucose sensor can function when implanted in the bloodstream of a dog for a period of several months and has the potential to operate for longer periods.

The success of the sensor as an intravenous implant in animals and the apparent biocompatibility suggest the possibility for this site to be used for chronic implantation in humans. A catheter-like sensor that can be easily introduced into the vena cava, retrieved and replaced every 3 to 6 months with a non-surgical procedure may be clinically useful. This application raises a concern about patient safety: there is the possibility of clot formation or vascular wall damage. However, it is well-documented that certain pacemaker leads and chronic drug delivery catheters implanted in the superior vena cava in humans can be biocompatible and are relatively innocuous [32, 33]. This sensor is ready for industrial development and implementation in humans may be relatively easily justified. Lag-free intravenous blood glucose recording may become the 'gold standard' and the most useful approach for control of insulin pumps.

\section{The state of the art}

Priority must now be given to consummation of the most advanced sensor approaches. There are no fundamental technical barriers to the use of oxygenbased sensors for short- and long-term intravenous applications and, given an effective industrial commitment, these applications could be available within a few years. The peroxide-based sensor also has some promise, mainly for short-term applications. Both the oxygen-based and peroxide-based sensors may eventually be useful in some capacity as subcutaneous implants, but more focused research is needed on the physiology of sensor-tissue interactions. Certain long-term intravenous sensors may actually be closer to clinical introduction and should be addressed more aggressively. Electrochemical sensor principles are much farther ahead than other principles.

Nevertheless, introduction of the sensor is not proceeding rapidly because of a subtle problem: the intention of industry appears to be introduction of subcutaneous short-term sensors first, then long-term sensors. This interest is a logical result of the substantial manufacturing talents available in industry and the potentially lucrative market for disposable sensors. However, as explained above, the present limitations of subcutaneous sensors are not related to manufacture, but rather to an insufficient understanding of physiologic aspects of sensor operation in tissues. Confident application of subcutaneous sensors requires a better understanding of the relevant physiological issues and a consensus in the research community on solutions to the remaining questions. The questions of understanding subcutaneous sensor function in tissue are best addressed in a university setting where a broader range of talents is available, and funded from public sources to foster objectivity. However, public funding agencies have often mistakenly held that sensor development is sufficiently advanced that further public support is not necessary. Meanwhile, development of the more straightforward long-term intravenous sensor has received less emphasis, even though a totally implantable sensor may be preferable to many potential users.

\section{Conclusions}

It is time for a consensus of investigators, funding agencies and device manufacturers that leads to a 
more rapid implementation of advances in glucose assay technology. The hope is that great strides in the treatment of diabetes associated with improvements in glucose assay will mark future anniversaries of the discovery of insulin.

A cknowledgements. This work was funded by grants from the Juvenile Diabetes Foundation International, the Whitaker Foundation, and the National Institutes of Health.

\section{References}

1. Gough D, Armour JC (1995) Development of the implantable glucose sensor: what are the prospects and why is it taking so long? Diabetes 44: 1005-1009

2. Marble A (1973) Laboratory procedures useful in diagnosis and treatment. In: Marble A, White P, Bradley RF, Krall LP (eds) Joslin's diabetes mellitus. Lea \& Febiger, Philadelphia, pp 191-208

3. Cooppan R (1994) General approaches to the treatment of diabetes. In: Kahn CR, Weir GC (eds) Joslin's diabetes mellitus. Lea \& Febiger, Philadelphia, pp 397-403

4. The Diabetes Control and Complications Trial Research Group (1993) The effect of intensive treatment of diabetes on the development and progression of long-term complications in insulin-dependent diabetes mellitus. N Eng J Med 329: 977-986

5. Blakeslee S (1994) Program to cut risks of diabetes surprisingly fails to lure patients. NY Times, February 28, p A1, C3

6. Lingenfelser T, Renn W, Sommerwerck U et al. (1993) Compromised hormonal counterregulation, symptom awareness, and neurophysiological function after recurrent short-term episodes of insulin-induced hypoglycemia in IDDM patients. Diabetes 42: 610-616

7. Clark LC Jr (1972) A family of polarographic enzyme electrodes and the measurement of alcohol. Biotech Bioeng 3: 377-394

8. Abel P, Muller A, Fischer U (1984) Experience with an implantable glucose sensor as a prerequisite of an artificial beta cell. Biomed Biochim Acta 43: 577-588

9. Shichiri M, Asakawa N, Yamasaki Y, Kawamori R, Abe H (1986) Telemetry glucose monitoring device with needletype glucose sensor: a useful tool for blood glucose monitoring in diabetic individuals. Diabetes Care 9: 298-301

10. Turner RFB, Harrison DJ, Rajotte RV, Baltes HP (1990) A biocompatible enzyme electrode for continuous in vivo glucose monitoring in whole blood. Sens Actuat B 1: 561564

11. Moatti-Sirat D, Capron F, Poitout V et al. (1992) Towards continuous glucose monitoring: in vivo evaluation of a miniaturized glucose sensor implanted for several days in rat subcutaneous tissue. Diabetologia 35: 225-230

12. Johnson KW, Mastrototaro JJ, Howey DC et al. (1992) In vivo evaluation of an electroenzymatic glucose sensor implanted in subcutaneous tissue. Biosens Bioelectron 7: 709-714

13. Gilligan BJ, Shults MC, Rhodes RK, Updike SJ (1994) Evaluation of a subcutaneous glucose sensor out to three months in a dog model. Diabetes Care 17: 882-887

14. Ward WK, Wilgus ES, Troupe JE (1994) Rapid detection of hyperglycaemia by a subcutaneously-implanted glucose sensor in the rat. Biosens Bioelectron 9: 423-428

15. Kerner W, Kiwit M, Linke B, Keck FS, Zier H, Pfeiffer EF (1993) The function of a hydrogen peroxide-detecting electroenzymatic glucose electrode is markedly impaired in human subcutaneous tissue and plasma. Biosens Bioelectron 8: 473-482

16. Moatti-Sirat D, Velho G, Reach G (1992) Evaluating in vitro and in vivo the interference of ascorbate and acetaminophen on glucose detection by a needle-type glucose sensor. Biosens Bioelectron 7: 345-352

17. Tse PHS, Gough DA (1986) Time-dependent inactivation of immobilized glucose oxidase and catalase. Biotech Bioengin 29: 705-713

18. Updike SJ, Hicks GP (1967) The enzyme electrode. Nature 214: 986-988

19. Gough DA, Leypoldt JK, Armour JC (1982) Progress toward a potentially implantable enzyme-based glucose sensor. Diabetes Care 5: 190-198

20. Armour JC, Lucisano JY, McKean BD, Gough DA (1990) Application of chronic intravascular blood glucose sensor in dogs. Diabetes 39: 1519-1526

21. Gough DA, Lucisano JY, Tse PHS (1985) A two-dimensional enzyme electrode sensor for glucose. Anal Chem 57: 2351-2357

22. Cui W, Ostrander LE, Lee BY (1990) In vivo reflectance of blood and tissue as a function of light wavelength. IEEE Trans Biomed Engin 37: 632-639

23. Fischer U, Ertle R, Abel P et al. (1987) Assessment of subcutaneous glucose concentration: validation of the wick technique as a reference for implanted electrochemical sensors in normal and diabetic dogs. Diabetologia 30: 940945

24. Moussy F, Harrison DJ, Rajotte RV (1994) A miniaturized nafion-based glucose sensor: in vitro and in vivo evaluation in dogs. Internatl J Artif Organs 17: 88-94

25. Claremont DJ, Sambrook IE, Penton C, Pickup JC (1986) Subcutaneous implantation of a ferrocene-mediated glucose sensor in pigs. Diabetologia 29: 817-821

26. Shults MC, Rhodes RK, Updike SJ, Gilligan BJ, Reining WN (1994) A telemetry-instrumentation system for monitoring multiple subcutaneously implanted glucose sensors. IEEE Trans Biomed Engin 41: 937-942

27. Shichiri M, Kawamori R, Yamasaki Y, Hakui N, Abe H (1982) Wearable-type artificial pancreas with needle-type glucose sensor. Lancet 2: 1129-1131

28. Pickup, JC, Claremont DJ, Shaw GW (1993) Responses and calibration of amperometric glucose sensors implanted in the subcutaneous tissue of man. Acta Diabetologica 30: $143-148$

29. Poitout V, Moatti-Sirat D, Reach G, Zhang Y, Wilson GS, Lemonnier F, Klein JC (1993) A glucose monitoring system for on line estimation in man of blood glucose concentration using a miniaturized glucose sensor implanted in the subcutaneous tissue and a wearable control unit. Diabetologia 36: 658-663

30. Gough DA, Armour JC, Lucisano JY, McKean BD (1986) Short-term in vivo operation of a glucose sensor. Trans Amer Soc Artif Intern Organs 32: 148-150

31. McKean BD, Gough DA (1988) A telemetry-instrumentation system for chronically implanted glucose and oxygen sensors. IEEE Trans Biomed Eng 35: 526-532

32. Lau YR, Gillette PC, Buckles DS, Zeigler VL (1993) Actuarial survival of transvenous pacing leads in a pediatric population. Pace 16: 1363-1367

33. Torramade JR, Cienfuegos JA, Hernandez JL et al. (1993) The complications of central venous access systems: a study of 218 patients. Eur J Surgery 159: 323-327 